\title{
Influence Factors on Outdoor Visual Landscape Satisfaction of Large-Scale Commercial Buildings in Winter City
}

\author{
Ziyuan Luo ${ }^{1,2, *}$, Hong Leng ${ }^{1,2}$ \\ ${ }^{1}$ School of Architecture, Harbin Institute of Technology, Harbin, China \\ ${ }^{2}$ Key Laboratory of Cold Region Urban and Rural Human Settlement Environment Science and Technology, Ministry of Industry and \\ Information Technology, Harbin, China
}

Email address:

luoziyuan-hit@qq.com (Ziyuan Luo),hitlaura@hit.edu.cn(Hong Leng)

${ }^{*}$ Corresponding author

To cite this article:

Ziyuan Luo, Hong Leng. Influence of Titanium Carbide on the Three - Body Abrasive Wear Behaviour of Glass-Fabric Reinforced Epoxy Composites. International Journal of Architecture, Arts and Applications. Vol. 4, No. 3, 2018, pp. 52-59. doi: 10.11648/j.ijaaa.20180403.14

Received: November 5, 2018; Accepted: December 12, 2018; Published: December 27, 2018

\begin{abstract}
The quality of outdoor visual landscape of large-scale commercial buildings in winter cities needs to be improved urgently, and residents' evaluation opinions are the important basis for countermeasures. In this paper, visual landscape images of large-scale commercial buildings were collected and were analyzed by Segnet decoder to obtain the proportion of each image. A questionnaire survey was also conducted on the satisfaction of residents' visual landscape based on greening, neatness, building recognizability, and visual comfort. The results show that the degree of satisfaction in winter is much lower than that in summer. By Analyzing the correlation between subjective satisfaction evaluation and objective factor composition, results show that the greening satisfaction is positively correlated with the visible green index, the neatness satisfaction is positively correlated with high-purity color, and negatively correlated with advertising and snow coverage. The building recognizability has a significant negative correlation with visible green index and a positive correlation with landscape arts. The visual comfort is positively correlated with the sky and high purity color and negatively correlated with snow coverage. Relevant countermeasures are put forward based on these results, such as increasing the visible green index, using high purity color, unifying advertising, increasing iconic landscape arts, and so on. The study also pointed out that users of large-scale commercial buildings and their outdoor environment are mostly women and the young.
\end{abstract}

Keywords: Visual Landscape, Environmental Satisfaction, Winter City, Quantitative Evaluation, Image Decomposition

\section{Introduction}

With the gradual improvement of urban residents' life quality in China, improving the quality of urban space has become an important research topic. Large-scale commercial buildings are important nodes for attracting residents to carry out leisure activities, and their outdoor environment is an integral part of public space. Its outdoor visual landscape directly reflects the urban landscape, residents' life quality, and residents' behavioral activities. Besides, the special climatic conditions of the winter city have an inevitable impact on the visual landscape. On the one hand, the distinct seasonal landscape and the unique snow resources in winter bring a distinctive visual experience. On the other hand, winter also weakens the green landscape and residents' visual experience. Residents' shopping, social and leisure activities in the winter significantly depend on large-scale commercial buildings and their outdoor environment. A good outdoor visual landscape helps attract residents to engage in related activities. Residents' evaluation of the visual landscape will serve as a guideline of the outdoor environment and an important basis for refined development [1].

Research on the visual landscape has been quite rich, involving the street environment, urban environment, and the rural landscape. Tang and Long used SegNet decoder to summarize the influencing factors of the streetscape and to measure the quality of streetscape with subjective evaluation [2]. Xu Jian et al. used MATLAB to analyze the landscape 
elements of the industrial park and combined the satisfaction survey to evaluate the landscape environment of the industrial park [3]. M. Arriaza et al. evaluated the visual quality of agricultural landscapes, pointing out that visual quality increased with the increase of artificial factors [4]. The outdoor environment of commercial buildings is also studied. Eun-Heui Lee et al. studied the green landscape around the commercial buildings in Seoul and believed that there should be at least one-meter wide green area in the outdoor of commercial buildings [5]. N Xing and KWM Siu explored the attractiveness of outdoor public space to consumers and the promotion of commercial activities [6]. However, existing visual landscape studies considered less about seasonal factors, especially winter in winter cities. Fu Zongchi et al. analyzed the impact of snowscape on visual comfort [7]. In addition to snow, seasonal differences in other factors may also affect the satisfaction of visual landscapes in winter cities, such as trees. The outdoor visual landscape of large-scale commercial buildings in winter cities is complex, and the influence of the constituent elements still needs further study.

Therefore, this paper took Harbin, a typical winter city in China, as an example. By collecting visual landscape images of large-scale commercial buildings, we obtained the proportion of each image with Segnet decoder. Using these images, a questionnaire survey was also conducted on residents' satisfaction of visual landscape. By analyzing the correlation between objective image composition and subjective image score, influence factors of residents' visual landscape satisfaction in winter and summer could be found.

\section{Method}

The main method is to calculate the proportion of each component by decomposing the visual landscape image and to investigate residents' satisfaction with the visual landscape image by questionnaire, then analyze the relationship between objective component composition and subjective satisfaction evaluation.

\subsection{Screen Subjects}

Based on relevant regulations, this study defined large-scale commercial buildings as a centralized layout with a building area of over $20,000 \mathrm{~m}^{2}$ and carries out various business activities and service supply. According to this, 6 large-scale commercial buildings in Harbin were screened, with different locations considered to ensure the representativeness of samples.

\subsection{Acquire Outdoor Visual Landscape Images}

Large-scale commercial buildings often occupy a certain plot of land independently. Therefore, this study defines the outdoor of large-scale commercial buildings as space enclosed by roads, natural boundaries, and the nearest building boundary.

In the process of field research, it is necessary to pre-select the sampling position of the captured image, clarify the sampling method of the visual landscape image, and the principle of screening and processing visual landscape images.

The method of selecting image sampling points refers to the systematic sampling method [8,9] and stratified sampling method [10] in the study of the visible green index. The boundary line of the outdoor environment of the building can be divided into linear and non-linear types. In order to accurately obtain an image with a non-linear boundary, it is necessary to set a sampling point at the sight turn. The linear boundary is relatively less affected and can be evenly distributed at intervals of $50 \mathrm{~m}$. For sampling points less than $30 \mathrm{~m}$ apart, the sampling point at the sight turn is preferentially retained. For boundary that was too close to the building, sampling points were placed on the opposite side of the road, as shown in Figure 1.

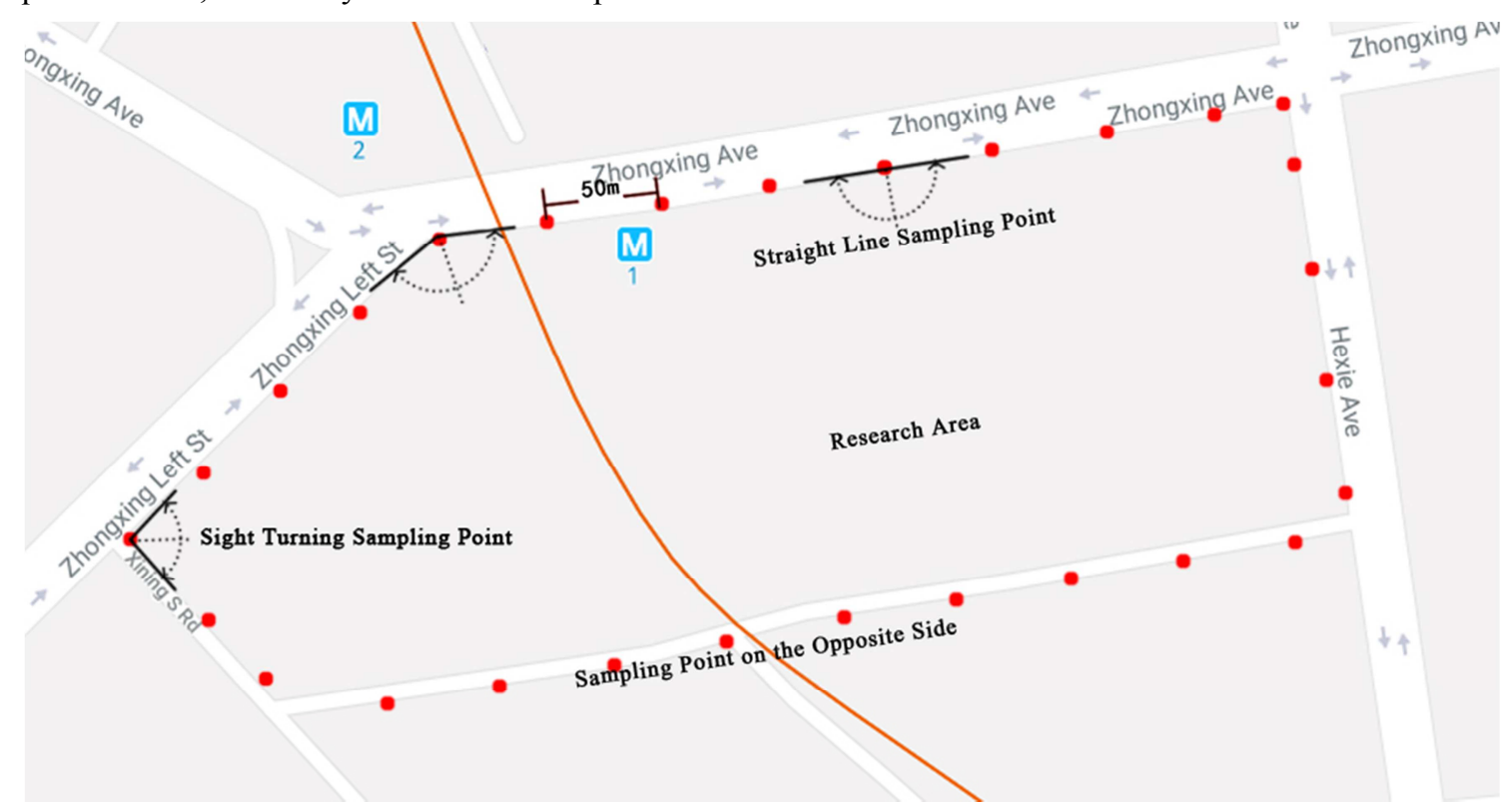

Figure 1. Sample Point Selection and Image Capture Method. 
The method of photoing the visual landscape image is determined to be centered on the sampling point, and the camera is horizontally turned from one side of the boundary line to the other side at a height of 1.6 meters, and the large-scale commercial building is always positioned as the main body of the image, as shown in Figure 1. Images were taken between 9:00 am and 4:00 pm on the weekend with a clear vision between December and January, to minimize the influence of glare, rain, and snow.

The principle of screening visual landscape image mainly involves three aspects. First, remove images with serious occlusion, overexposure, blur distortion, and similar content. Then, adjust images in darkness to ensure the maximum clarity. Considered the outdoor environment type and landscape content of the image, this study finally selected 10 representative visual landscape images each in winter and summer.

\subsection{Analysis of Objective Elements}

Deep convolutional neural networks (DCNNs) is currently the most advanced method of semantic image segmentation [11], and the fully convolutional networks (FCNs) have been used as standard tools for neural network software. The Segnet developed by the University of Cambridge is a software platform built by a fully convolutional networks with coders and decoders [11, 12], which can identify 12 types of elements such as sky, buildings, poles, road markings, roads, pavement, trees, signs, fences, cars, bicycles and pedestrians, as shown in Figure 2. After testing a variety of indoor and outdoor RGB images, Segnet decomposition images are considered to be reliable [2] and have been widely used.

The outdoor environment of large-scale commercial buildings is more specific and complex than others, as sculptures and other landscape arts are always arranged to enhance the environmental quality and attract consumers. A variety of landscape arts have gone beyond Segnet's recognition range. Therefore, this study identified the landscape arts in the image artificially. Snow in winter images also has a strong impact on the accuracy of the Segnet decomposition. Therefore, this study used Photoshop software to correct the Segnet analysis results, as shown in Figure 3. Winter cities often use high-purity bright colors to adjust the visual landscape [13], so the high-purity color was taken as one of the influencing factors. As shown in Figure 4, high-purity color in the images was extracted by Photoshop without the interference of the sky. In addition, this study used the ratio of trees as the visible green index. The visible green index refers to the proportion of green plants in the line of sight of the human eye. Compared with the greening rate and green coverage rate, it can dynamically and intuitively reflect the difference of visual landscape between winter and summer. Taking the proportion of the identified poles as the proportion of infrastructure, it is mainly considering that the design of large-scale commercial buildings' outdoor environment pursues a clean and beautiful appearance, and infrastructure exposed to the visual range is mostly poles such as electric poles and light poles.

The proportion of pixels occupied by each component in the corrected Segnet result is counted by Photoshop software.
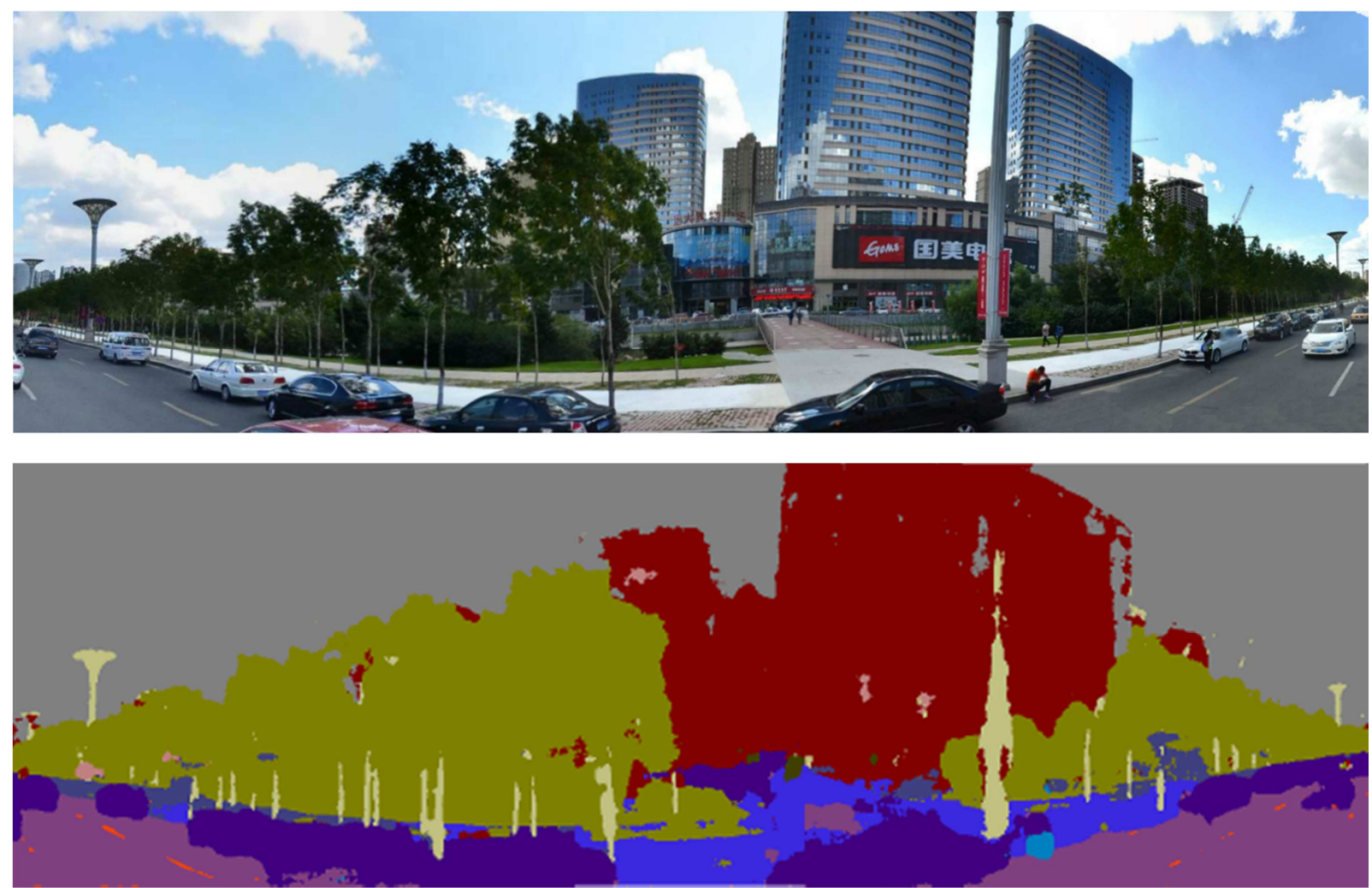

Figure 2. Image before and after Segnet decomposition. 

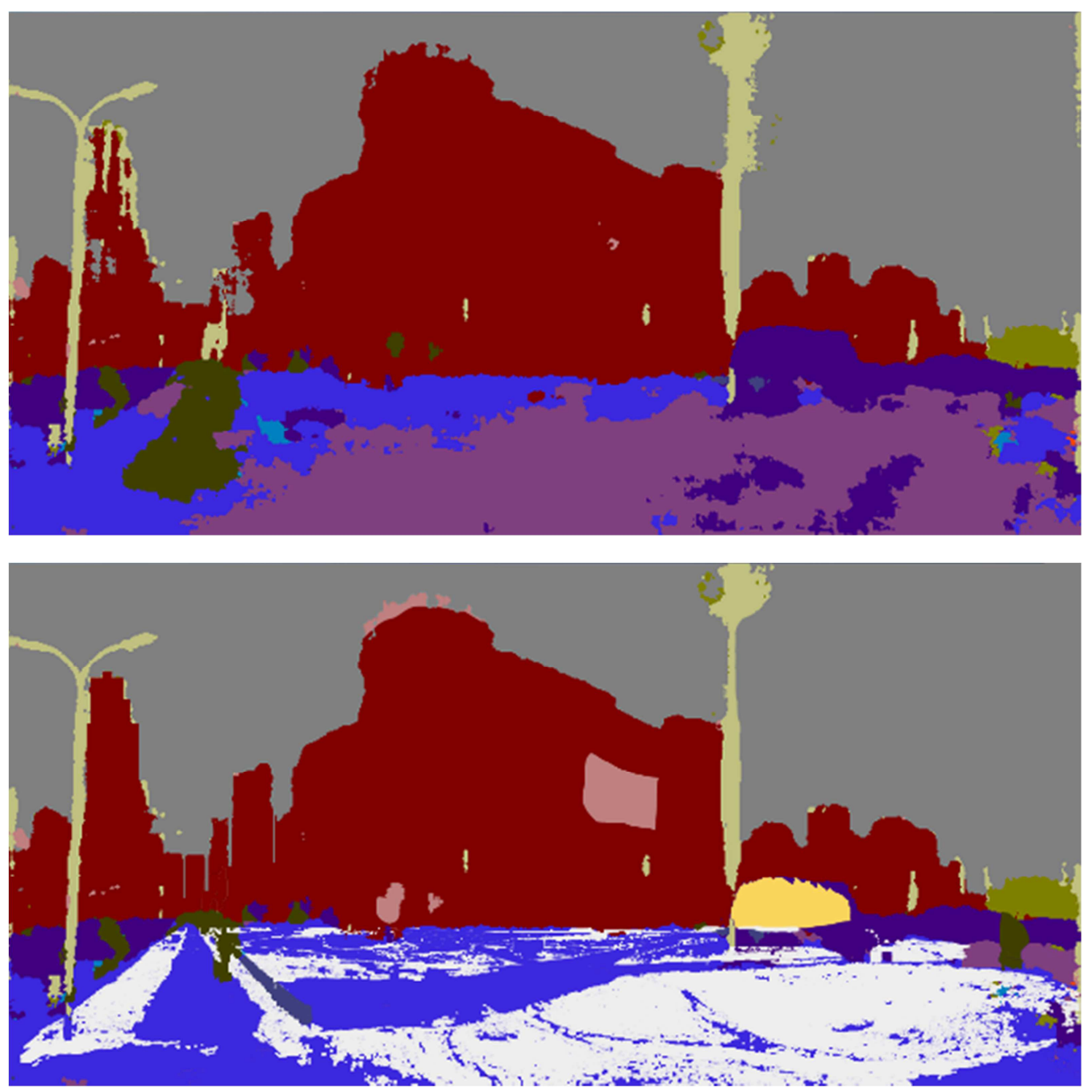

Figure 3. Segnet decomposition image before and after correction.
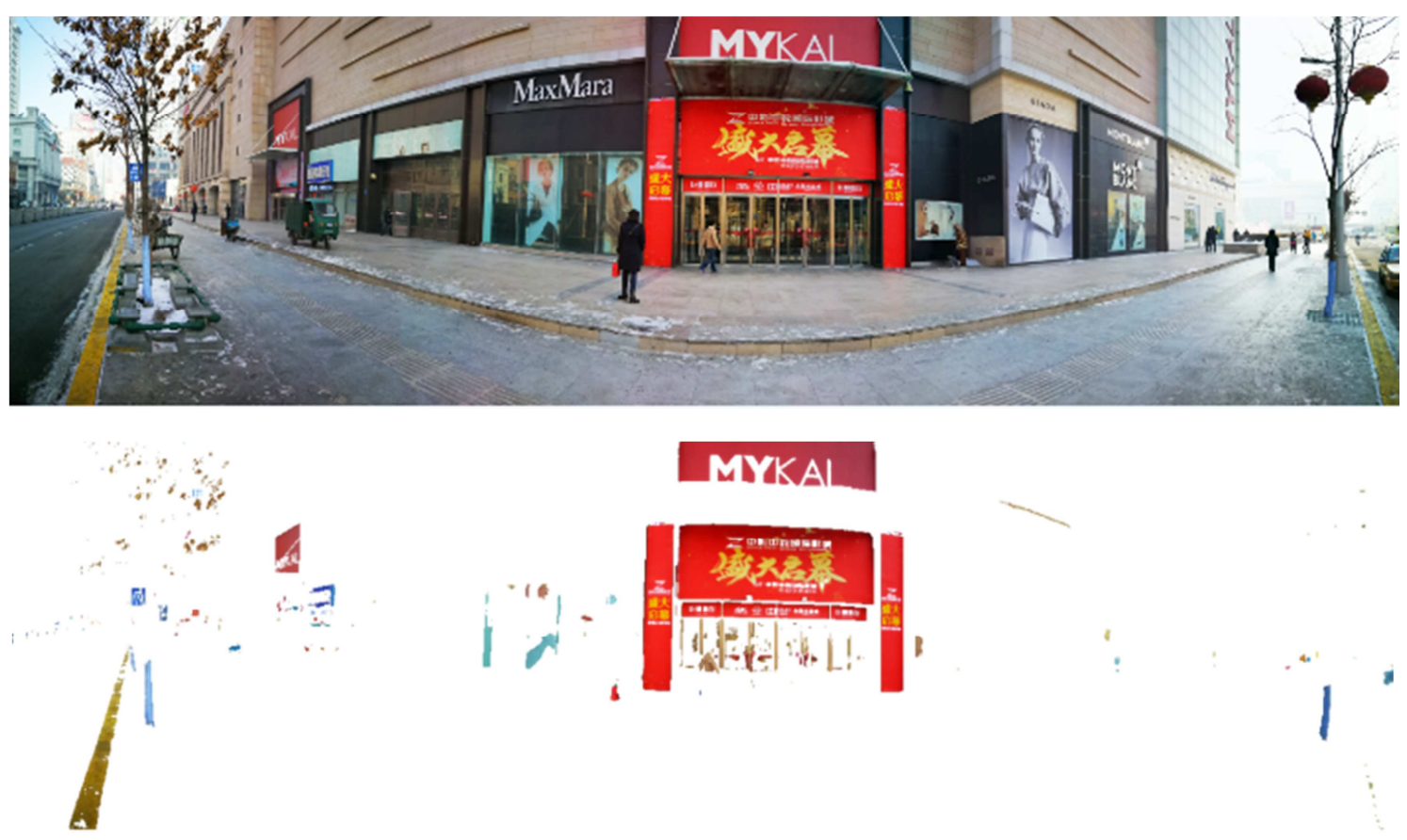

Figure 4. Image before and after extracted high-purity colors with Photoshop. 


\subsection{Subjective Satisfaction Evaluation}

Images can be used instead of real scenes for evaluation [14, 15]. According to the characteristics of the outdoor visual landscape and the elements of image decomposition, the satisfaction of the outdoor visual landscape is divided into four indicators: greening, neatness, building recognizability and visual comfort. The greening is mainly for the natural environment, and the neatness is mainly for the artificial environment. For large-scale commercial buildings and their outdoor environment, residents can accurately identify their building functions, and even specific buildings are particularly important. Therefore, building recognizability should be considered as one of the indicators. Visual landscape satisfaction is influenced by objective elements and their attributes, as well as subjective feelings. Therefore, visual comfort is considered an indicator of visual psychology. The residents evaluated the satisfaction of the same picture from the above four aspects. The satisfaction here mainly refers to: the residents compare images with their expects and judge whether the outdoor visual landscape meets their expectations. Dissatisfaction is 0 point, satisfaction is 1 point.

A large number of color pictures in the questionnaire makes it difficult to print, this survey was done in the form of electronic questionnaires. According to the formula of sample capacity, the minimum sample size was estimated with an error tolerance of $5 \%$. The results showed that 246 valid questionnaires need to be recovered to achieve the accuracy requirements. In the actual investigation process, 266 questionnaires with 250 valid questionnaires were collected, which met the minimum requirement. The effective recovery rate of the questionnaire was $93.98 \%$. According to the reliability of the Cronbach's $\alpha$ reliability formula, the reliability $\alpha=0.943$, which was over 0.7 . The reliability of the survey data has reached the requirements of the following study.

\section{Results}

\subsection{Basic Characteristics}

According to the results of the questionnaire, a basic understanding of gender composition and age characteristics of the interviewee can be formed. The ratio of male to female was about 1:4, indicating that women use far more large-scale commercial buildings and their outdoor environment than men. Therefore, the needs of women should be fully considered. In terms of overall age distribution, the composition of large-scale commercial buildings and their outdoor environment is young. The youth group of 18 to 40 years old is the main body of large-scale commercial buildings and their outdoor environment, accounting for $86 \%$; the middle-aged population of 40 to 60 years old accounts for $10 \%$; teenagers under 18 years old account for a relatively small proportion (4\%), this may be related to weak social needs and heavy school workload. The youth group has a rich social activity and prefer a high-quality lifestyle that focuses on leisure and entertainment. The lack of people over the age of 60 is due to the fact that the small number of questionnaires recovered have been eliminated due to poor quality, which also reflects elder people's limited use of outdoor environment due to their inconvenient mobility.

\subsection{Correlation Analysis of Objective Element Composition and Subjective Satisfaction Evaluation}

The average scores of the 20 images are summarized as shown in Table 1, and the images with even numbers are winter images. The total score of the image is the sum of the scores of greening, neatness, building recognizability and visual comfort. It can be divided into 5 levels from the highest 4 points to the lowest 0 points. The average score of the image is concentrated in the range of 1 to 3 points, and is mostly 2 to 3 points, accounting for $65 \%$. It can be considered that the respondents are basically satisfied with the outdoor visual landscape of large-scale commercial buildings in Harbin. Summer images score significantly better than winter images, indicating that residents' satisfaction with the outdoor visual landscape in winter is much lower than that in summer, and the visual landscape in winter needs to be improved.

Table 1. Summary of the average scores of each image resident score.

\begin{tabular}{|c|c|c|c|c|c|}
\hline No. & Greening & Neatness & Building recognizability & Visual comfort & Sum \\
\hline 1 & 0.052 & 0.92 & 0.868 & 0.428 & 2.268 \\
\hline 2 & 0.092 & 0.948 & 0.936 & 0.728 & 2.704 \\
\hline 3 & 0.868 & 0.808 & 0.46 & 0.78 & 2.916 \\
\hline 4 & 0.292 & 0.792 & 0.732 & 0.472 & 2.288 \\
\hline 5 & 0.484 & 0.568 & 0.448 & 0.716 & 2.216 \\
\hline 6 & 0.188 & 0.252 & 0.504 & 0.264 & 1.208 \\
\hline 7 & 0.736 & 0.416 & 0.604 & 0.404 & 2.16 \\
\hline 9 & 0.264 & 0.224 & 0.552 & 0.268 & 1.308 \\
\hline 10 & 0.172 & 0.292 & 0.472 & 0.256 & 1.192 \\
\hline 11 & 0.564 & 0.636 & 0.616 & 0.512 & 2.328 \\
\hline 12 & 0.236 & 0.568 & 0.644 & 0.752 & 2.2 \\
\hline 13 & 0.448 & 0.856 & 0.696 & 0.648 & 2.648 \\
\hline 14 & 0.432 & 0.68 & 0.768 & 0.612 & 2.492 \\
\hline 15 & 0.528 & 0.828 & 0.66 & 0.644 & 2.66 \\
\hline
\end{tabular}




\begin{tabular}{|c|c|c|c|c|c|}
\hline No. & Greening & Neatness & Building recognizability & Visual comfort & Sum \\
\hline 17 & 0.772 & 0.796 & 0.784 & 0.708 & 3.06 \\
\hline 18 & 0.144 & 0.504 & 0.744 & 0.452 & 1.844 \\
\hline 19 & 0.944 & 0.856 & 0.372 & 0.74 & 2.912 \\
\hline 20 & 0.292 & 0.304 & 0.508 & 0.36 & 1.464 \\
\hline
\end{tabular}

Pearson correlation analysis was used to study the correlation between the composition of various elements and satisfaction evaluation, as shown in Table 2. The correlation between the composition of various elements in the winter and summer images and the satisfaction evaluation is analyzed separately, as shown in Tables 3 and 4. According to the study, the scope of the outdoor environment of large-scale commercial buildings is defined, and the influence of roads and road markings is not considered. Following results can be discovered:

The greening satisfaction in summer is significantly and positively correlated with the visible green index and negatively correlated with the building. There is no correlation between winter and various elements. However, overall, there is still a positive correlation between greening satisfaction and visible green index, and a negative one with pavement.

The neatness satisfaction is negatively correlated with advertising logo in summer and fence and snow coverage in winter. Overall, the satisfaction of neatness is positively correlated with high-purity color, and negatively correlated with advertising logo and snow coverage.

The building recognizability in summer is positively correlated with the building and negatively correlated with the visible green index. In winter, it is only positively related to landscape arts. Overall, there is still a significant negative correlation between building recognizability and visible green index.

The visual comfort in summer is positively correlated with the sky and high purity color and negatively correlated with architectural and advertising logo. In winter, it is positively related to the landscape. Overall, visual comfort is positively correlated with the sky and high purity color, and negatively correlated with snow coverage.

There was a positive correlation between overall summer satisfaction and high purity color, and a negative correlation with the advertising logo. Overall winter satisfaction was positively correlated with landscape arts and negatively correlated with snow coverage. On the whole, overall satisfaction is positively correlated with high-purity color and negatively correlated with advertising logo and snow coverage.

Table 2. Correlation analysis of the proportion of each element in the decomposition of the image and the score $(* p<0.05 ; * * p<0.01)$.

\begin{tabular}{llllll}
\hline & Greening & Neatness & Building recognizability & Visual comfort & Sum \\
\hline sky & 0.152 & 0.272 & 0.121 & $0.446^{*}$ & 0.35 \\
building & -0.262 & -0.093 & 0.304 & -0.413 & -0.208 \\
infrastructure & 0.085 & 0.032 & -0.06 & -0.09 & 0.008 \\
Pavement & $-0.545^{*}$ & 0.061 & 0.243 & -0.113 & -0.198 \\
Visible green index & $0.728^{* *}$ & 0.225 & $-0.529 * *$ & 0.347 & 0.396 \\
Advertising logo & -0.107 & $-0.587^{* *}$ & -0.285 & -0.438 & $-0.497 *$ \\
Fence & 0.021 & -0.039 & -0.188 & 0.091 & -0.026 \\
Vehicle density & 0.313 & -0.176 & -0.284 & -0.075 & -0.024 \\
Pedestrian density & -0.077 & -0.126 & -0.001 & -0.311 & -0.183 \\
bicycle & 0.115 & 0.207 & 0.055 & 0.153 & 0.198 \\
Landscape art & -0.085 & 0.207 & 0.181 & $0.521^{*}$ & 0.253 \\
Snow coverage & -0.436 & $-0.583^{* *}$ & -0.168 & $-0.532^{*}$ & $-0.645^{* *}$ \\
High purity color & 0.384 & $0.572^{* *}$ & 0.166 & $0.591^{* *}$ & $0.635^{* *}$ \\
\hline
\end{tabular}

Table 3. Summary of the average scores of each image resident score.

\begin{tabular}{|c|c|c|c|c|c|}
\hline & Greening & Neatness & Building recognizability & Visual comfort & Sum \\
\hline sky & -0.249 & 0.029 & 0.426 & 0.241 & 0.17 \\
\hline building & 0.119 & 0.098 & -0.182 & -0.116 & -0.026 \\
\hline infrastructure & -0.067 & 0.106 & -0.196 & -0.315 & -0.128 \\
\hline Pavement & 0.262 & 0.221 & 0.058 & 0.228 & 0.24 \\
\hline Visible green index & 0.191 & -0.197 & -0.397 & -0.459 & -0.32 \\
\hline Advertising logo & 0.078 & -0.38 & -0.469 & -0.181 & -0.349 \\
\hline Vehicle density & 0.056 & -0.374 & -0.463 & -0.552 & -0.474 \\
\hline Pedestrian density & -0.174 & 0.326 & -0.169 & -0.178 & 0.004 \\
\hline bicycle & 0 & 0 & 0 & 0 & 0 \\
\hline Landscape art & -0.278 & 0.603 & $0.690 *$ & $0.856^{* *}$ & $0.701 *$ \\
\hline Snow coverage & -0.216 & $-0.749 *$ & -0.453 & -0.624 & $-0.712^{*}$ \\
\hline High purity color & -0.42 & 0.512 & 0.468 & 0.42 & 0.424 \\
\hline
\end{tabular}


Table 4. Summary of the average scores of each image resident score.

\begin{tabular}{|c|c|c|c|c|c|}
\hline & Greening & Neatness & Building recognizability & Visual comfort & Sum \\
\hline sky & 0.366 & 0.506 & -0.113 & $0.680 *$ & 0.612 \\
\hline building & $-0.692 *$ & -0.338 & $0.658^{*}$ & $-0.800 * *$ & -0.589 \\
\hline infrastructure & 0.035 & -0.028 & -0.014 & -0.101 & -0.032 \\
\hline Pavement & -0.59 & 0.459 & 0.419 & -0.051 & -0.004 \\
\hline Visible green index & $0.733^{*}$ & 0.24 & $-0.649 *$ & 0.497 & 0.469 \\
\hline Advertising logo & -0.254 & $-0.861 * *$ & -0.109 & $-0.764 *$ & $-0.804 * *$ \\
\hline Fence & -0.131 & 0.17 & -0.071 & 0.304 & 0.085 \\
\hline Pedestrian density & 0.019 & -0.39 & 0.083 & -0.388 & -0.266 \\
\hline bicycle & -0.048 & 0.213 & 0.123 & 0.121 & 0.145 \\
\hline Landscape art & -0.12 & -0.07 & -0.142 & 0.33 & -0.028 \\
\hline Snow coverage & 0 & 0 & 0 & 0 & 0 \\
\hline High purity color & 0.54 & 0.551 & -0.022 & $0.676^{*}$ & $0.751 *$ \\
\hline
\end{tabular}

\subsection{Countermeasures for Outdoor Visual Landscape Improvement of Large-scale Commercial Buildings in Winter Cities}

Correlation analysis shows that there are differences in the factors affecting the satisfaction of winter and summer, and should be targeted when proposing improvement strategies. When conditions are limited, the overall satisfaction should be given the highest priority, as shown in Table 5.

Table 5. Countermeasures for outdoor visual landscape improvement of large-scale commercial buildings in winter cities.

\begin{tabular}{|c|c|c|c|}
\hline & Priority overall strategy & Additional strategy in summer & Additional strategy in winter \\
\hline Greening & $\begin{array}{l}\text { Increase the visible green index } \\
\text { Properly reduce the proportion of pavement } \\
\text { Use high purity color }\end{array}$ & Rich vegetation varieties & $\begin{array}{l}\text { Increase evergreen vegetation } \\
\text { Plastic plants temporary decoration }\end{array}$ \\
\hline Neatness & $\begin{array}{l}\text { Clear snow } \\
\text { Unified advertising logo }\end{array}$ & & Remove the fence \\
\hline $\begin{array}{l}\text { Building } \\
\text { recognizability }\end{array}$ & $\begin{array}{l}\text { Reduce tall trees, use shrubs and ground cover } \\
\text { Increase the space between plantings } \\
\text { Increase sky visibility }\end{array}$ & & Iconic landscape art \\
\hline Visual comfort & $\begin{array}{l}\text { Clear snow } \\
\text { Use high purity color } \\
\text { Clear snow }\end{array}$ & Unified advertising logo & Iconic landscape art \\
\hline Sum & $\begin{array}{l}\text { Use high purity color } \\
\text { Unified advertising logo }\end{array}$ & & Increase landscape arts \\
\hline
\end{tabular}

Timely cleaning up snow, increasing the use of high-purity colors, and unifying advertising logos are the top priorities for improving the outdoor visual landscape of large-scale commercial buildings in winter cities. The glare reflected by the snow can cause irritation to the human eye and even cause snow blindness in severe cases. The use of high-purity colors can be adjusted to improve the monotonous visual environment and enrich residents' visual experience. Different styles of advertising logos can be dazzling, causing visual discomfort, by unifying advertising logos can effectively improve visual comfort. In addition, the layout of the iconic landscape can further improve the visual landscape in winter. The iconic landscape arts can effectively improve the building recognizability in winter. According to Kevin Lynch's imagery theory, good building recognizability makes commercial buildings become nodes and even landmarks in the city, attracting more attention from residents. Increase the chances of residents using commercial buildings and the outdoor environment. Successful landscape design will also become synonymous with the outdoor environment. On the other hand, the visual landscape in winter is more monotonous than in summer, and the working mechanism of the human eye determines that human sight needs to focus with a certain point of interest, otherwise it will cause psychological anxiety. Distinctive landscape arts can attract residents' attention, enhance visual comfort and increase the utilization rate of commercial buildings and their outdoor environments, and encourage consumption activities.

Further improvement of the visual landscape should be targeted based on four aspects: greening, neatness, building recognizability and visual comfort. In order to improve the satisfaction of greening, the greening rate should be increased, vegetation variety should be enriched, and the visual monotony brought by large-area square pavement should be avoided. Specifically, part of square pavement can be replaced with ground cover plants or be divided with plants. During the winter, increase the use of evergreen vegetation or temporarily decorate with plastic plants should be considered. It is worth noting that the visible green index is negatively correlated with the building's recognizability, but it does not mean that increasing the building's visibility will reduce the visible green index. The recognizability of large-scale commercial buildings can be enhanced by increasing the space between plantings; or appropriately reducing tall trees, using shrubs and ground cover plants. In order to improve the satisfaction of neatness in winter, in addition to cleaning up the snow, fences should be removed to create an open visual psychological experience, promote 
residents' use of large-scale commercial buildings and their outdoor environment, and encourage commercial vitality. By increasing the visibility of the sky, to avoid the psychological pressure of buildings over-obstructing the sky can further enhance the visual comfort.

\section{Conclusion}

This study aimed at the outdoor visual landscape of large-scale commercial buildings in winter cities studied the correlation between subjective evaluation and objective factor composition of greening, neatness, building recognizability, visual comfort, and overall satisfaction.

According to the characteristics of the respondents, it can be found that there are far more female users than male in large-scale commercial buildings and their outdoor environment. The composition of users is young, mostly concentrated between the ages of 18 and 40 . The visual preferences of female groups and youth groups should be fully considered in landscape design.

According to the subjective satisfaction evaluation, residents' overall satisfaction with the outdoor visual landscape in summer is better than winter, the visual landscape in winter needs to be improved.

According to the correlation analysis between the objective factor composition and the subjective satisfaction evaluation, there are differences in influence factors in winter and summer. The countermeasures to improve the overall satisfaction should be the highest priority. These findings can provide a reference for further improvement of the outdoor visual landscape in winter cities.

\section{Acknowledgements}

Supported by the Sub-project of the "13th Five-Year Plan" National Key R\&D Program "Research on Green Public Building Planning and Landscape Design Adapting to Cold Climate" (2017YFC0702306).

\section{References}

[1] Daniel T C. Whither scenic beauty? Visual landscape quality assessment in the $21 \mathrm{st}$ century [J]. Landscape \& Urban Planning, 2001, 54 (1):267-281.

[2] Tang Jingxian, Long Ying. Metropolitan Street Space Quality Evaluation: Second and Third Ring Of Beijing, Inner Ring Of Shanghai [J]. Planners, 2017, 33 (2):68-73. (In Chinese).
[3] Xu Jian, Kang Jian, Shao Long. Subjective Evaluation of Landscape Environment in Industrial Parks [J]. Chinese Landscape Architecture, 2015, 31 (9):95-99. (In Chinese).

[4] Arriaza M, Cañas-Ortega J F, Cañas-Madueño J A, et al. Assessing the visual quality of rural landscapes [J]. Landscape \& Urban Planning, 2004, 69 (1):115-125.

[5] Lee E H, Jang H K, Ahn G Y. The Use of Landscape Greenery Surrounding Commercial Buildings in Seoul [J]. Journal of the Korean Institute of Landscape Architecture, 2008, 36 (5).

[6] Xing N, Siu K W M. Outdoor Public Environment of Shopping Malls: A Balance between Consumption and Social Practice. [J]. International Journal of the Constructed Environment, 2011.

[7] Fu Zongchi, Qiu Hongfei, Li Yingbin. Research on the Visual Comfort of Snowscape in Cold Area [J]. Chinese Landscape Architecture, 2017, 33 (7):113-116. (In Chinese).

[8] Green Vision Survey and Greening Residue Review Business Summary Report (Summary) [EB/OL]. Japan: Kyoto. (2014-03)[2015-08-10]. http://www.city.koto.lg.jp/seikatsu/douro/CIG/midori/ryokusir itu/file/tyousagaiyou.pdf.

[9] Li X, Zhang C, Li W, et al. Assessing street-level urban greenery using Google Street View and a modified green view index [J]. Urban Forestry \& Urban Greening, 2015, 14 (3):675-685.

[10] Central District Green State Survey (4th) [EB/OL]. Japan. (2005-03)[2015-08-16].

http://www.city.chuo.lg.jp/kankyo/midori/Zittaityosa.files/330 -007.pdf.

[11] Badrinarayanan V, Kendall A, Cipolla R. Seg Net: A Deep Convolutional Encoder-Decoder Architecture for Scene Segmentation. [J]. IEEE Transactions on Pattern Analysis \& Machine Intelligence, 2017, PP (99):1-1.

[12] Marmanis D, Schindler K, Wegner J D, et al. Classification With an Edge: Improving Semantic Image Segmentation with Boundary Detection [J]. 2016.

[13] Leng Hong, Guo Enzhang, Yuan Qing. Research on the Design Countermeasures of Climate City [J]. Urban Planning, 2003, 27 (9): 49-54. (In Chinese)

[14] Barrosoaabaa F L. Dealing with landscape fuzziness in user preference studies: Photo-based questionnaires in the Mediterranean context [J]. Landscape \& Urban Planning, 2012, 104 (3):329-342.

[15] Ives C D, Kendal D. Values and attitudes of the urban public towards peri-urban agricultural land [J]. Land Use Policy, 2013, 34 (12):80-90. 